der im. Sinne günstiger immunbiologischer Wirkung zu erklären wäre. Eingehendste Prüfung hatte ein negatives Ergebnis. Es war nirgends eine Wirkung vorhanden, die nicht vollkommen ungezwungen durch den Pneumothorax allein zu erklären gewesen wäre. Es zeigt sich sogar im Gegenteil, daß gerade besonders günstige Wirkungen, nämlich Dauerheilungen, nur bei den trockenen Pneumothoraces und solchen mit kleinen Randexsudaten zu verzeichnen waren. Je höher das Exsudat steigt und besonders je mehr es Temperatursteigerungen hervorruft, desto mehr sinkt die Aussicht auf einen besonders günstigen Verlauf der Tuberkulose und des Verfahrens. Daraus muß man ganz allgemein ableiten, daß das Exsudat als der Erfolg einer Pleuritis nichts Günstiges sein kann.

Das Wesentliche bei der Beurteilung der prognostischen Bedeutung der Exsudate scheint mir das zu sein, daß abgesehen von wenigen die ungünstige Wirkung sich nicht sofort, sondern erst nach längerer Zeit häufig erst bei Abschluß des Verfahrens entwickelt, daß das Exsudat ständig die Aussicht, das Ideal der Pneumothoraxtherapie, nämlich die vollkommene Wiederausdehnungs- und Wiederatmungsfähigkeit der Lunge, zu erreichen, verschlechtert. Das ist für den Kranken besonders gefahrvoll. Ich verstehe darunter die Bildung von Schwarten, den Eintritt der Obliteration der Pleurahöhle evtl. vor Heilung der Lungentuberkulose, Retraktion der betreffenden Thoraxhälfte und nicht zuletzt die Belastung des rechten Herzens. Dies alles wird sich, abgesehen von den Randexsudaten bei allen größeren Exsudaten, mag ihre Entwicklung mit oder ohne Fieber vor sich gegangen sein, einstellen. Daß es sich bei meinen Kranken um 102 diesbezügliche Fälle bzw. 59\% handelt, ist ein nachdrücklicher Hinweis auf die Wichtigkeit. Diese Tatsache fällt auch bei der Frage des doppelseitigen Pneumothorax, der an sich so außerordentlich wünschenswert wäre, sehr ins Gewicht. Hier könnten, wenn wirklich doppelseitige obliterative Prozesse auftreten, ernste Störungen von seiten des rechten Herzens die Folge sein.

Die Exsudate, die in den Gruppen $D$ bis $F$ zusammengefaßt sind: $4 \mathrm{I}$ Fälle $=24 \%$ sind ohne weiteres ungünstig aufzufassen. Bei ihnen steht eben die entzündliche Komponente ganz im Vordergrund. Ich möchte besonders darauf hinweisen, daß von den 6 Empyemen nur eins durch Einriß an der Lungenoberfläche entstanden ist, und alle andern sich allmählich aus Exsudaten entwickelt haben. Bei den Exsudaten der Gruppe D bis F tritt $z$ den, wie oben ausgeführt, mehr in der Zukunft liegenden Störungen die augenblickliche der stürmischen Entwicklung hinzu. Hier ist der Eindruck augenfällig und muß gegenüber den spärlichen Mitteilungen aus der Literatur ganz besonders betont werden. Die späteren Störungen stellen sich auch bei diesen wesentlich schneller ein. Umgekehrt liegt es bei den fieberlosen größeren Exsudaten. Diese machen zunächst wenig oder gar keine Erscheinungen. Das ist aber gerade das Heimtückische für den Kranken. Die Folgen sind in jedem einzelnen Falle nie absehbar und werden spätestens in dem sowieso schon kritischen Augenblick der Wiederausdehnung der Lunge akut. Die Eigenschaft eines größeren Exsudates als Füllsel zu dienen, kann nicht als etwas Erstrebenswertes gelten, da man bei der herrschenden Eigenart der Exsudate, sich unversehens zu resorbieren oder schnell zu steigen, mit unangenehmen Anderungen stets zu rechnen hat. Der Gedanke, daß man bei ihnen nicht so oft nachfüllen muß, bringt keinen wirklichen Vorteil, da der Kranke doch regelmäßig nachzuuntersuchen ist, und die Nachfüllung bei geeigneter Technik keine Belästigung bedeutet.

Die fieberlosen kleinen Randexsudate - es gibt auch vereinzelt solche mit schweren fieberhaften Begleiterscheinungen - sehen sehr harmlos aus und sind es zunächst auch meist. Wie weit bei ihnen später adhäsive Prozesse an den Pleuren sich einstellen, wird außerordentlich schwer zu beurteilen sein. Ganz harmlos dürfen sie nie beurteilt werden, weil sich aus ihnen stets ein größeres entwickeln kann. Daher ist häufige röntgenologische Kontrolle besonders bezüglich des rechten Zeitpunktes der Nachfüllung sehr am Platze.
Daß bei solchen Arbeiten mit hohem Druck evtl. ein schnelles Steigen eintreten kann, ist ohne weiteres zuzugeben.

Wenn ich so zum Schlusse komme, daß das Exsudat, das im ganzen genommen in $100 \%$ auftritt, schrittweise mit seiner immer größeren Ausdehnung immer unwillkommener wird, und besonders, wenn Fieber damit verbunden ist, stets eine schwere Komplikation bedeutet, deren Folgen häufig erst spät zum Ausdruck kommen, so soll dies Urteil doch, man könnte es herauslesen, nichts gegen den Pneumothorax selbst besagen. Diesem bleibt seine in geeigneten Fällen gan $\dot{z}$ vorzügliche Wirkung unbenommen. Nur in der Richtung der Indikationsstellung $\mathrm{mu} B$ diese Beurteilung der Exsudate von Bedeutung sein. Man wird, so wie es auch dem Zug der Zeit in der Therapie augenblicklich entspricht, leichte Fälle, denen man bei Sanatoriumskur Aussicht auf Heilung geben kann, ausschließen und überhaupt das Pneumothoraxverfahren im allgemeinen erst anwenden, wenn der Versuch eines Heilstättenaufenthaltes negativ verlaufen ist. Denn das muB man sich vor Augen halten, man setzt jeden Kranken, der sich dem Pneumothorax unterwirft, ohne daß wir bisher eine Handhabe zum Eingreifen dagegen haben, der Möglichkeit schwerer Komplikationen von seiten des Exsudates aus, die entscheidenden Einfluß auf den Ablauf der Tuberkulose und damit auf das Leben des Kranken haben können.

Lite r a tur: Aumeurlere, Journal de Pract. 1921, Nr, 28. ARNSPERGER, ref. Tuberkul.-Zentralbl. 17, 10o. - BoAs, New Yorls med. Journ. 192 I, Nr. I I. - BRAUER, Ber. üb. d. Wiesbadener Kongr. Ig21. - Burnand, Paris m. d. I92 I, Nr. i7. - Burnand, Tuberculosis I92 I, Nr. 4. - CantanI, ref. Tuberkul.-Zentralbl. 16, 228. - CRocket, Edinburg, med. Journ. 25, Nr. 2. 1920. - Deist, Brauers Beiträge. - DEYcke, Lehrb. d. Tuberkul. I92 I. - vaN Driessche, Scalpel i92I, Nr. 4I. - Dumarest, Ann. de mécl. 8, Nr. 4. I920/21. -- Dumarest, Policlinico 28, H. 7. I92 I. Frey, Der künstl. Pneumothorax ig21. - GugGenheimer, Therapie der Gegenwart IgIg, H. 2. - HANSEN, Med. Klinik r920, Nr. 48. - Harms, Zeitschr. f. ärztl. Fortbildung igr9, Nr. 16. HedINGER, Zeitschr. f. angew. Anat. 6. I920. - JAgUerod, Rev, med. de la Suisse rom. I9I2, I 53. - JAVA, Rivista I912, Nr. I9. - JeHN, Ber. üb. d. Wiesbadener Kongr. r92I. - Joss, Brauers Beitr, 46. I92 I. - KomlHaAs, Med. Korrespondenzblatt I9IO, 755; I9I6, I2 I. - LeschKe, Münch. med. Wochenschr. 1920, II $35 .-$ Lux, Progr. med. 1920, Nr. 2 I. - Niederhäusern, Schweiz. med. Wochenschr. - PEERs, Californ. State Journ. of med. I9, Nr. 8. 1921. - Penato, Il Morgagni 7 . Nr. Io. - Rickmann, Brauers Beitr. 46. 1920. - Renon, Paris. med. 1921, Nr. I. - Rivers, Lancet 199. I920. - SAMson, Klin. therapeut. Wochenschr. I92I, Nr. 15-18. - Sauerbruch, Chirurg. der Brustorgane 1920. Sauerbruch, Münch. med. Wochenschr. 1921, Nr. 9. - SaugMANN, Lancet I920, Nr. I4. - SACHSTORPH, Ugeskrift f. Laeger I921, Nr. 22. - Schwenkenbecher, Med. Klinik i920, Nr. 20. Stivelmann, Ref. Tuberkul.-Zentralbl. I6, 354. - StivelmanN, Journ. of the Amer. med. Assoc. I92 I, Nr. I. - TIDEström, Brauers Beitr. Suppl. I92 I. - Vogeizang, ref. Tuberkul.-Zentralbl. I5, 395.

\section{DIE LEHRE VOM KRANKHAFT GESTEIGERTEN INTRAUTERINEN DRUCK UND DIE ENTSTEHUNG DER ANGEBORENEN DEFORMITÄTEN.}

\author{
Von \\ Priv.-Doz. Dr. Alfred Schubert. \\ Aus der Chirurgischen Universitäts-Klinik zu Königsberg i. Pr. \\ (Direktor: Professor Dr. M. KIRSCHNER.)
}

Wer es heute unternimmt, für das Zustandekommen det angeborenen Deformitäten mechanische Vorgänge als alleinige Ursache abzulehnen und insbesondere die so bequeme und für alles brauchbare Theorie von der krankhaften Steigerung des intrauterinen Druckes in ihrer ätiologischen Bedeutung erheblich einzuschränken, muß auf vielfachen Widerspruch gefaßt sein. Der Angriff von WeIL (Klin. Wochenschr. I922, Nr. 25, S. I 260) gegen meine Anschauungen von der Entstehung der Schiefhalserkrankung und von der der $\mathrm{Ge}$ burtslähmung kam daher nicht unerwartet. Bedauerlich ist es nur, daß WEIL seinen Artikel schrieb, ehe die von mir án- 
gekündigte ausführlichere und inzwischen erschienene Arbeit im Druck herauskam ${ }^{1}$ ); manche Erörterung wäre damit unnötig geworden, da ihnen durch meine dortigen Ausführungen bereits der Boden entzogen ist. Bedauerlich ist es auch, daß gerade $W_{E I L}$, der in der Frage der Geburtslähmung durch seine Hypothese von der im intrauterinen Leben bereits vorhandenen Plexusschädigung manche veraltete Anschauung über Bord warf, nun doch so prinzipiell an der mechanischen Ursache dieser und anderer Deformitäten festhält.

WEIL behauptet, meine Theorie von der zentralnervösen Plexũsschädigung im Sinne eines Bildungsdefektes widerspräche den Tatsachen. Er hebt besonders drei Punkte hervor, auf die ich kurz eingehen will:

I. Bildungsfehler heilen nicht spontan; da aber ,so viele Fälle von Armlähmung" heilen, sei meine Theorie falsch. Wo sind die vielen geheilten Fälle? Von den zuletzt von ValentiN vor einem Jahre mitgeteilten sechs Fällen ist noch jahrelang nach der Geburt kein einziger geheilt, wobei ich hoffe, daß WEIL unter ,Heilung" nicht etwa durch Gewöhnung, Übung, Mitbenutzung anderer Muskeln herbeigeführte rein funktionelle Besserung versteht. Alle zeigen deutliche Veränderungen des knöchernen und muskulären Schulterapparates und werden sie voraussichtlich auch zeitlebens - trotz eingeleiteter Behandlung - behalten. WEIL selbst teilte seine Fälle bisher nicht genauer mit; wenn es aber in einem seiner Fälle zu einer Fraktur des Schlüsselbeins kam, die WeIL auch entsprechend meiner Anschauung auf neuropathische Knochenveränderung zurückführt, so fehlt mir der Glaube an die spontane Heilbarkeit einer derartigen Plexusschädigung. An Spontanheilung von Plexusschädigung glaube ich nur bei den Fällen, bei denen wirklich isoliert der Plexus im Moment der Geburt im Sinne der Erbschen Lähmung verletzt wurde. Diese Fälle gibt es natürlich, ich habe sie auch ausdrücklich (mit Nachuntersuchungen) in meiner ausführlicheren Arbeit erwähnt. Ob diese Fälle häufig oder - wie ich es annehme seltener sind, ist für uns Chirurgen schwer zu entscheiden, da uns diese Fälle naturgemä $B$ selten zugehen. Für uns handelt es sich um die ätiologische Klarstellung derjenigen Fälle, die frïhzeitig oder bereits im Moment der Geburt deutliche Knochenund Muskelveränderungen aufweisen. Offenbar haben VALENTIN und WEIL bei der Aufstellung ihrer Theorien, ebenso wie ich, derartige Fälle im Auge gehabt. Die Spontanheilung dieser Fälle muß erst beschrieben werden; wir haben sie nicht beobachtet.

2. Ich versuchte gerade den Nachweis zu erbringen, daß zur Erklärung der gesamten neurotrophischen Störung ein isolierter Druck auf den Erbschen Punkt nicht ausreicht und daß man daher eine weitere zentralwärts gelegene Störung annehmen muß, will man nicht zu dem mißlichen Ausweg greifen, eine Multiplizität von Schädigungen anzunehmen. Daß aber eine Narbenbildung am Plexus durch , die immer zahlreicher gewordenen Eingriffe bei Kindern mit Armlähmung" in neuester Zeit häufiger nachgewiesen wurde, deckt sich weder mit den Angaben der Literatur noch mit WeILs eigenen Anschauungen, der sich vor einem Jahre sehr reserviert gegen jede therapeutische Plexusfreilegung aussprach. Da Mitteilungen über Operationsbefunde sehr spärlich sind und recht verschiedenartige Deutung zulassen, hätte WEIL, wenn ihm inzwischen derartig bedeutungsvolle Funde geglückt sein sollten. jeden neu erhobenen anatomischen Befund möglichst genau beschreiben sollen.

3. Die anatomische Veränderung des Kopfnickermuskels bei der Schiefhalserkrankung der einfachen Muskelatrophie bei der Geburtslähmung gleichzusetzen, ist mir nie eingefallen. Allerdings nehme ich an, daB beide anatomisch sehr verschiedenen Muskelveränderungen und die Veränderungen noch zahlreicher anderer Gewebsteile, die dem Krankheitsherd benachbart sind oder ihm zugehören (Knochen, Gefäße usw.), letzten Endes auf nervöse Einflüsse zurückzuführen sind.

Der Frage der Heredität habe ich hinsichtlich der Geburtslähmung keine allzu große Bedeutung beigelegt. Dagegen be-

2) Ubrigens sind beide Arbeiten in der Dtsch. Zeitschr. f. Chirurg. 167 und 170 und nicht in Bruns' Beiträgen erschienen. tonte ich die meines Erachtens außerordentlich wichtige gegenseitige Kombination der verschiedenen angeborenen Deformitäten. Da ein Teil dieser Deformitäten zweifellos vererbbar ist, $\mathrm{kam}$ ich in diesem Zusammenhang auch auf die Erblichkeit der Geburtslähmung zu sprechen und erwähnte die wenigen, hierfür maßgebenden Fälle.

Für äußerst gekünstelt halte ich den Unterschied, den WEIL zwischen "Schulterblatthochstand" und "Schulterhochstand" macht. Nur dann wäre ihm recht zu geben, wenn der "Schulterhochstand" bei der Geburtslähmung wirklich die von ihm behauptete günstige Prognose hätte. Valentin z. B. führt unter seinen sechs Fällen allein vier mit deutlichem, durch mehrere Jahre unverändert bestehendem Schulterhochstand an, und ähnliche Beobachtungen sind nicht selten. Und wie erklärt WEIL die wiederholt festgestellte Verkleinerung des Schulterblattes in all seinen Dimensionen? Ist das auch eine Folge des krankhaft gesteigerten intrauterinen Drucks?

Gerade in dieser Kombination der Geburtslähmung mit dem echten Schulterblatthochstand, der auch nach WeILs Ansicht nicht durch vermehrten intrauterinen Druck zustande kommt, und darüber hinaus in der Kombination mit Bauchmuskeldefekten (WEIL), Schiefhals, Hüftverrenkung und anderem mehr sehe ich einen der Hauptgründe, warum ich für die Ätiologie der Geburtslähmung die krankhafte Steigerung des intrauterinen Drucks ablehne.

Gibt es den Anhängern der Theorie von dem alles verursachenden krankhaft gesteigerten intrauterinen Druck nicht $z u$ denken, daß für immer mehr Deformitäten (Hüftverrenkung, KlumpfuB) diese rein mechanische Ursache unwahrscheinlich wird? Vielleicht ist es bezeichnend, daß die an und für sich recht wichtigen Röntgenbefunde von WARNEKROS und SIPPEL als stärkste Stütze dieser Theorie immer wieder herangezogen werden. Ich habe wiederholt betont, daß wir aus diesen Röntgenbefunden nur die fertige Tatsache der falschen Lage, nicht aber ihre Ätiologie entnehmen können. Solange wir aber nichts Sicheres über das Zustandekommen der normalen Geburtslagen wissen, ist es mehr als gewagt, aus pathologischen Zustandsbildern Schlüsse auf ihr Zustandekommen zu ziehen.

Wenn aber WeIL sagt: „Die Röntgenbilder haben die abnorme intrauterine Lage und Pressung der Schulter gegen den Hals bei Uterusenge unumstößlich bewiesen ", so bleibt unberücksichtigt, daß die Röntgenbilder wohl die abnorme intrauterine Lage, nicht aber eine (passive) Pressung beweisen können.

Ich halte die Anschauungen über die rein mechanische Entstehung der Geburtslähmung und der Schiefhalserkrankung für unvereinbar mit klinischen Tatsachen und kann ihnen nur sekundäre Bedeutung zumessen, insofern, als die primär aus sich heraus entstandene Deformität durch die in jedem Uterus vorhandene Beengung der Lage noch weiter verstärkt wird. Die Annahme einer krankhaften Steigerung des Uterusinnendruckes ist hierzu weder erforderlich noch wahrscheinlich; sie ist auch durch nichts bewiesen.

\section{EINE MIKROSPORIE-EPIDEMIE IN FRANKFURT a. M.}

Von

Dr. Georg Klein und Dr. C. Aliferis.

Aus der Dermatologischen Universitätsklinik zu Frankfurt a. M.

(Direktor: Geh. Med,-Rat Prof. Dr, K. HERXHEIMER.)

In den letzten Jahren wurden aus vielen Großstädten Mikrosporieepidemien beschrieben. Auch in Frankfurt a. M. konnten wir etwa seit Ende vorigen Jahres das Auftreten einer kleinen Epidemie beobachten. Wenn wir es nun unternehmen, diese Epidemie zu veröffentlichen, so geschieht es einerseits wegen des übereinstimmenden klinischen und kultu- 